\title{
Pulmonary Hypertension from Cardiorenal Sequelae in an 18-Year-0ld 46, XY Phenotypically Female Patient with Denys-Drash Syndrome: Case Report and Review of Literature
}

\author{
Mouhamed Nashawi MD*, Abdullah Ghali BS², Momin Hussain BS², David Momtaz BS' ${ }^{2}$, Aum Mhapankar \\ BS $^{2}$, Katie Struck BS ${ }^{2}$ and Robert Chilton DO FACC, FAHA, MACOI, FSCAI ${ }^{2}$
}

${ }^{1}$ Department of Internal Medicine, Baylor Scott \& White All Saints Medical Center, Texas, USA

${ }^{2}$ Division of Medicine-Cardiology, UT Health San Antonio, San Antonio, Texas, USA

*Corresponding author: Mouhamed Nashawi, Department of Internal Medicine, Baylor Scott \& White All Saints Medical Center, 1400 8th Avenue, Fort Worth, TX, 76104-4110.

Received Date: June 09,2021

Published Date: June 23,2021

\begin{abstract}
Denys-Drash Syndrome (DDS) is a rare cause of end-stage renal disease (ESRD) stemming from genetic mutations in the Wilms' tumor 1 (WT1) gene. Albeit uncommon, it and other congenital nephropathies (e.g., Frasier syndrome) may warrant a clinical workup of cardiovascular disease in symptomatic patients with cardiopulmonary deficits, as the latter is associated with renal dysfunction given the multifarious physiologic roles and nodes of action concerning the kidneys. These include not only serving as a site of neural and hormonal stimuli that affect systemic equilibrium, but the regulation of blood pressure and osmolality as well. The cardiorenal demise classically seen in later stages of chronic kidney disease (CKD) in relatively older adults may arise earlier in these patients. We report the case of an 18-year-old 46, XY phenotypic female with DDS who complained of increasingly nettlesome dyspnea. Initially, she presented with a low index of suspicion for diminished cardiopulmonary fitness. However, further clinical workup which included catheterization and cardiac imaging was significant for findings of pulmonary hypertension.
\end{abstract}

Key words: Pulmonary hypertension, Heart failure, Cardiorenal, Denys-drash, Echocardiography, Wilms

\section{Background}

Cardiorenal syndrome (CRS), defined by the Acute Dialysis Quality Initiative (ADQI), refers to a spectrum of clinical manifestations in which renal and cardiovascular physiology are disturbed due to pathologic dysfunction of one organ affecting the other [1]. CRS is not a sequential unidirectional process and may also include limitations in both organs that reaches a critical threshold (e.g., systemic deposition of amyloid light-chain amyloidosis) [2]. The ADQI have defined five subtypes for the delineation of CRS (Figure 1). Cardiovascular risk factors implicated in cardiorenal syndrome include older age, myocardial injury (manifested as troponin leak), and moderate to severe mitral valve regurgitation manifesting as reduced left ventricular ejection fraction $[3,4]$. CRS is associated with nephropathies of congenital onset in young adults. For example, nephropathy stemming from diabetes and acquired nephropathies such as post-infectious glomerulonephritis [5].

Cardiorenal syndrome in young adults with congenital nephropathy is commonly reported as rapid renal failure and systemic decline, or premature coronary artery disease [6]. Overlooked are defects in the great vessels of young adults with congenital nephropathy. Namely, pulmonary hypertension in 
the latter is of interest to the clinician managing patients with complicated cardiorenal syndrome of congenital origin. Mindfulness of this phenomenon can prove vital in the management of these patients.

Pulmonary hypertension from cardiorenal syndrome can be more discernable in these patients on hemodialysis, presenting with shortness of breath, dyspnea upon exertion, and decreased cardiopulmonary fitness. We report the case of congenital nephropathy secondary to Denys-Drash Syndrome (DDS) in an 18-year-old phenotypically female $46, \mathrm{XY}$ patient with symptoms concerning for dyspnea, later revealed to have pulmonary hypertension despite recent cardiac imaging portraying a less acute clinical picture.

We believe this to be the first report of pulmonary hypertension related to cardiorenal sequelae of DDS. Given the rarity of DDS in the literature in addition to the paucity of reports in the literature characterizing cardiorenal syndrome manifesting as acute pulmonary hypertension in the young adult with congenital nephropathy, reports of these encounters are valuable in informing providers and guiding the workup of pulmonary vasculopathy in those with nephropathy.

\section{Case Description}

An 18-year-old 46, XY phenotypically female patient with a past medical history of end-stage renal disease (ESRD) and remote surgical history significant for cadaveric right lower renal transplantation presented to our institution to explore eligibility for new renal transplantation after it was determined her original allograft of 11 years underwent chronic rejection. Her nephropathy was deemed to be secondary to congenital Denys-Drash syndrome, confirmed with a positive WT1 mutation upon genetic testing. Her renal function at the time warranted a Monday-Wednesday-Friday regimen of hemodialysis. Medications include amlodipine and lisinopril for their renoprotective properties, and cholecalciferol to help maintain mineral equilibrium in the setting of kidney disease. She was deemed to be an appropriate surgical candidate for transplantation pending appropriate medical clearance and monitoring. These included frequent electrocardiograms, transthoracic echocardiograms (TTE), cardiovascular stress testing, renal function tests, and examinations of arteriovenous fistula.

Initial TTE revealed moderate mitral valve regurgitation and pulmonary valve insufficiency (Figure 2). Two months later, subsequent TTE affirmed the absence of pulmonic stenosis or valvular lesions with mild pulmonary valve insufficiency and slight mitral valve dysfunction in the context of increased complaints of dyspnea upon exertion, fatigue, paroxysmal nocturnal, and orthopnea at hemodialysis appointments (Figure 3). A fivemonth TTE revealed exacerbation of heart failure with findings of newfound global hypokinesis noted in the cardiac septum and inferior wall with severe, grade III diastolic dysfunction (Figure 4). Mitral and tricuspid central annular dilatation with moderate to severe central mitral regurgitation and moderate central tricuspid regurgitation were noted. Ejection fraction was estimated to be $40 \%$, consistent with systolic heart failure with reduced ejection fraction. A PASP of $32 \mathrm{mmHg}$ with a CVP of $3 \mathrm{mmHg}$ was measured, warranting cardiac catheterization for assessment.

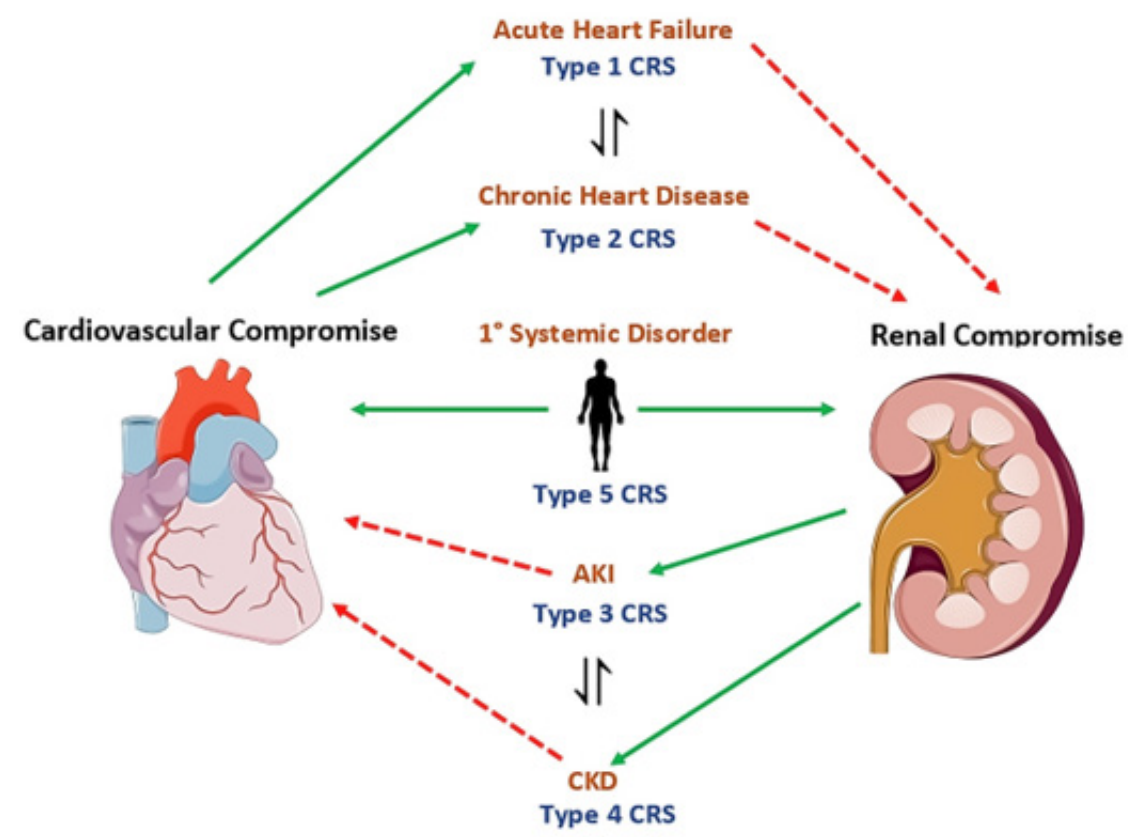

Figure 1: Subtypes of Cardiorenal Syndrome as defined by the AQDI. adapted from Servier

Medical Art by Servier, which is licensed under a Creative Commons Attribution 3.0 Unported License. 


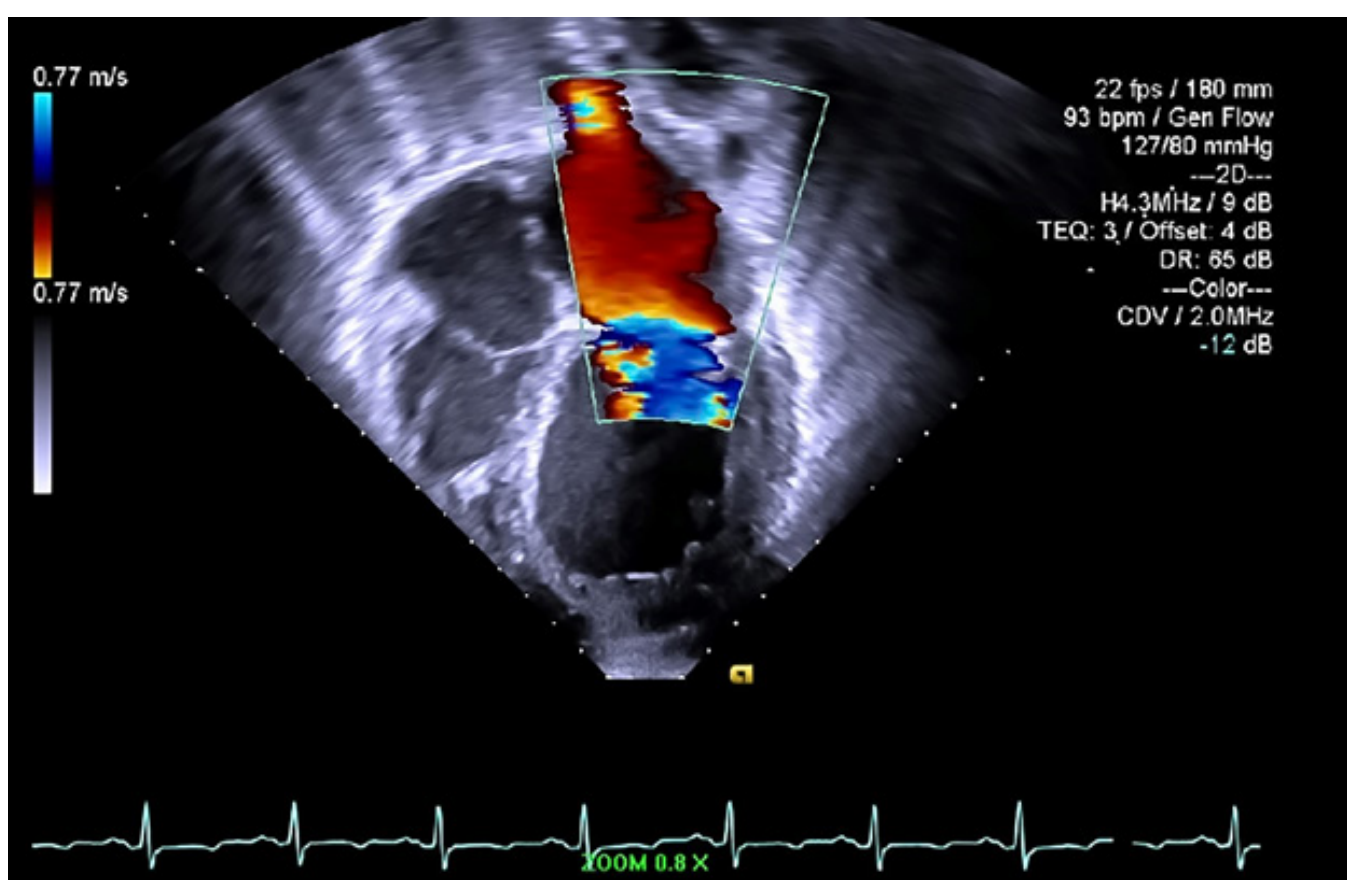

Figure 2: Initial TTE Showing Moderate Mitral Regurgitation and Pulmonary Valve Deficit.

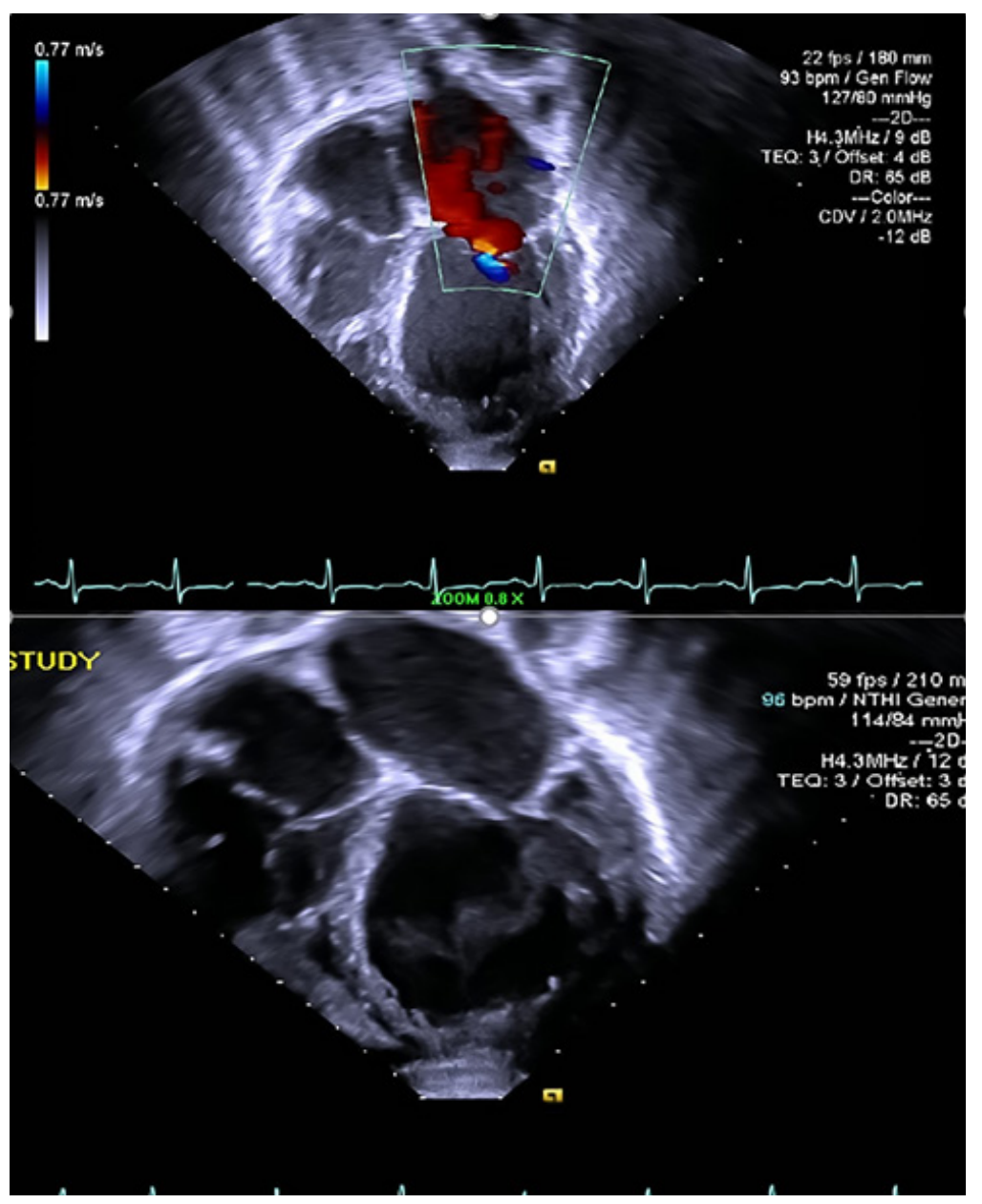

Figure 3: Two-Month Confirming Exacerbation of Pulmonic Valve Function. 


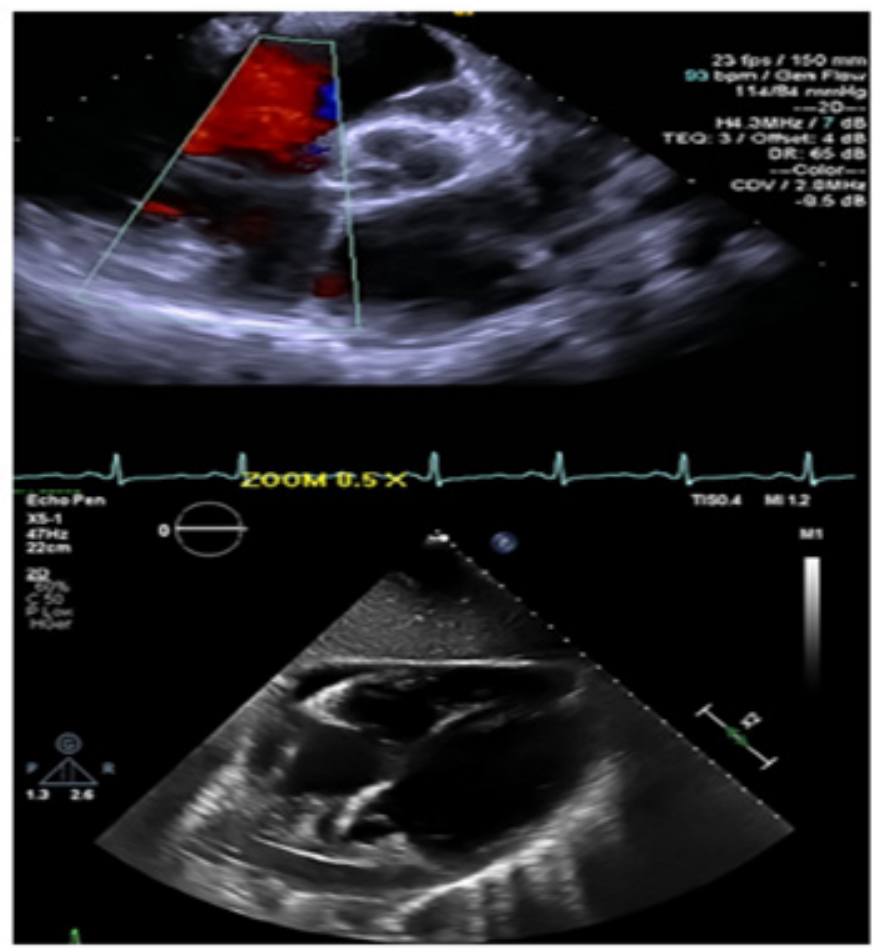

Figure 4: Five-month TTE Showing Severe Diastolic Dysfunction Indicative of Pulmonic Dysfunction.

Left heart catheterization (LHC) revealed normal heart morphology, coronaries, and vascular structure with a lack of tamponade with a pulmonary artery pressure of 54/26 $\mathrm{mmHg}$. This elevated pulmonary artery pressure was determined to be secondary to mixed pulmonary venous and arterial hypertension with the patient subsequently discharged to bed rest. Given these findings, the pulmonary hypertension in this patient is World Health Organization (WHO) Groups 2 and 5 (for systolic left ventricular dysfunction and ESRD, respectively). Due to the lack of evidence and risk factors for pulmonary arterial hypertension (WHO Group 1), there exists no benefit or indication for pulmonary vasodilators. Previous attempts at vasodilation use in this setting has resulted in a lack of statistically significant clinical improvement, even potentially causing harm. Recommendations for these patients are a goal of euvolemia through pharmacotherapy and lifestyle changes. Prospective renal transplantation would help reduce cardiorenal sequelae through improved control of neurohormonal regulation (modulation of renin-angiotensin-aldosterone-system), which would promote a healthier pulmonary vascular profile in this patient.

\section{Discussion}

Pulmonary hypertension (PH) is characterized by elevated blood pressure within the pulmonary vasculature. Common etiologies include increased sympathetic tone (e.g., stimulant use or hypoxia induced vasoconstriction) or mechanical impediments that prevent adequate blood flow in the heart or lungs, causing strain [7-9]. Systemic inflammatory disorders have also increasingly gained awareness in their role in the pathogenesis of pulmonary artery hypertension, possibly through the induction of reactive oxygen species that control the genetic expression of factors that reduce vascular pliability, namely endothelin-19. PH may also arise from dysfunctional great vessels development, which is present in DDS manifesting as patent ductus arteriosus and coarctation of the aorta [10]. While not studied in-depth in human subjects due to the high fatality rate in DDS, WT1 mutations in mice have been shown to alter epicardium formation, with these structural configurations implicated in cardiovascular disease [11]. Such alterations are due to disorders of epithelial-to-mesenchymal transformations within cardiomyocytes inhibiting adequate tissue differentiation, promoting a phenotype more conducive to disease. DDS is characterized by gonadal dysgenesis, nephropathy, and nephroblastoma from mutations in WT1, a transcription factor regulating the development of the urogenital system [12]. Commentary on WT1 in DDS is paramount in delineating the pathophysiology observed in our patient and bridging CRS with PH. WT1 is as a transcription factor cited in renin transcription and glomerular capillary formation [13]. Dysfunctional WT1 results in inadequate glomerular filtration of toxins, namely uremic toxins implicated in platelet dysfunction [14]. Exacerbating this problem is the lack of proper estrogen metabolism (as seen in DDS due to gonadal dysgenesis), resulting in further platelet dysfunction [15]. The aggregate effect of this dysfunction is diminished pulmonary vascular tone and subsequent PH through aberrant thrombosis 
regulation [16]. Furthermore, PH results in decreased oxygen flux, manifesting as the noted dyspnea in this case. Hypoxia has been known to induce mitochondrial damage, promoting the formation of reactive oxygen species (ROS) that contribute to cardiomyocyte hypertrophy with increased tension via NF- $\kappa$ B17. ROS also modulates nitric oxide activity with consequences in endothelial cell dysfunction, resulting in hemodynamic instability, irregular renal arterial in-flow, pulmonary arterial tone, and dysfunctional cardiovascular physiology $[17,18]$. By following this WT1 gene, we believe that our patient with DDS suffered from a type-4 CRS profile. Type-4 CRS is managed through renal transplantation to ameliorate renal dysfunction by correcting hypertension, as supported by guidelines investigating CRS [19].

An appraisal of the renal physiology and neuromodulation in the context of chronic disease gives an avenue for further characterization of our patient's presentation. ESRD is associated with the inability of nephrons to promote diuresis and fluid balance, which classically manifests as central congestion. Further compromise of the kidneys supports a physiologic equilibrium that is acclimated to the retention of metabolic toxins which directly serve as insults to the heart and kidney in cardiorenal syndrome as defined by KDIGO [20]. In normal physiologic circumstances, increased congestion caused by edema results in stretch of native mechosensory nerve fibers scattered throughout the abdominal and pelvic wall20. This leads to a phenomenon known as the renorenal reflex, by which activation of this system attenuates efferent renal sympathetic nervous system activity (ERSNA) [21,22]. Decreased ERSNA is associated with a higher threshold for $\alpha 1$-adrenoceptor activation. These receptors play a vital role in the activation of transporters in the proximal tubule that lead to a state of net sodium reabsorption. By decreasing their function, the renorenal reflex induces natriuresis and relief of central congestion. However, chronic kidney disease and ESRD is associated with dysregulation of the renorenal reflex [23,24].

In the case of a patient who has hampered kidney function such as in the case of our patient with DDS, a lack of renorenal reflex may cause $\alpha 1$-adrenoceptor activation to prevail. This imbalance of autonomic nervous system can be appreciated in the presence of these receptors within the smooth muscle lining pulmonary vasculature, and theories suggesting that overactivation of these receptors promotes vasoconstriction and long-term remodeling that promotes pulmonary hypertension $[25,26]$. In addition to promoting overactivity of $\alpha$ 1-adrenoceptors, increased ERSNA tone supports are associated with an increased expression and activity of $\beta 1$-adrenoreceptors [27]. The $\beta 1$-adrenoreceptors family are well accounted for in their tendency to promote pathologic overactivation of RAAS cascade, through increased renin. Multiple accounts in the literature have studied the association between $\beta 1$ adrenoreceptor tone and pulmonary hypertension via remodeling of the vasculature surrounding the lung parenchyma, which may have also prevailed in contributing towards the pulmonary hypertension observed in this patient with DDS [28-30]. Reports in the literature note the use of angiotensin converting enzyme inhibitors (ACEI) in the treatment of DDS [31]. The rationale for such an approach includes a reprieve of efferent arteriole constriction within the nephrons, which is associated with decreased ESRNA responses and attenuation of autonomic shifts that promote the aforementioned vasculopathies.

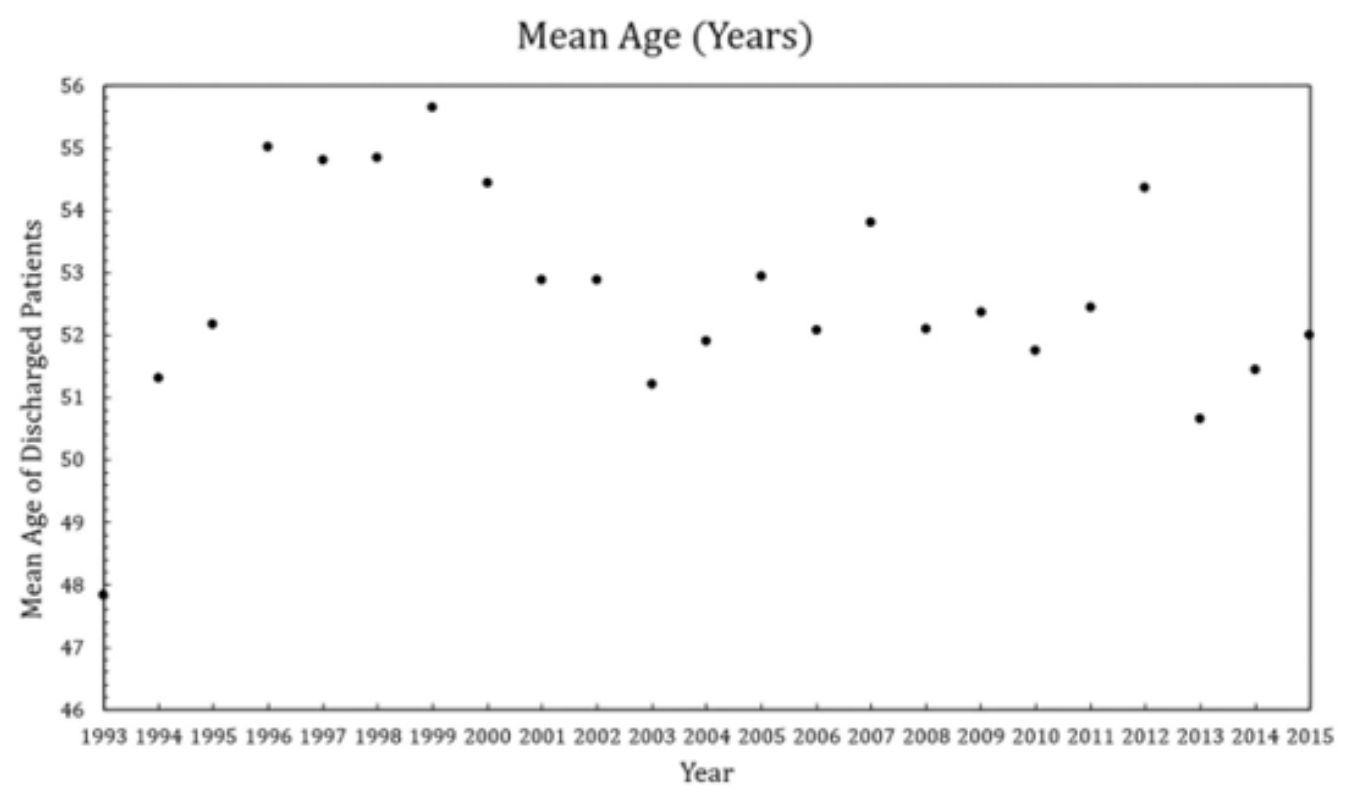

Figure 5: Mean Age of Discharged Patients with Pulmonary Hypertension Per National Inpatient Sampling Database (NIS). 
PH rarely presents in the hospital setting before the age of 20, as demonstrated from the National Inpatient Sample (NIS), a publicly available all-payer inpatient database containing information on hospital admissions, as the mean age of discharge for pulmonary hypertension in the United States is over 40-years (Figure 5) [32]. Badesch et. al studied the age of onset of PH in the general population in the Registry to Evaluate Early and Long-term PAH Disease Management (REVEAL) study, a multi-center study that recruited 2,967 patients with pulmonary hypertension, showing PH is most commonly diagnosed between 45 and 54 years of age [33]. The presenting signs of pulmonary hypertension include syncope, shortness of breath, and edema from perturbed blood and oxygen flow. Reports in the literature about pulmonary hypertension in patients with congenital nephropathy such as a Denys-Drash Syndrome (DDS) are scant. While these patients are evaluated for renal transplantation, as seen in this case, there exists the potential for lost proclivity in managing the cardiorenal sequelae of chronic renal failure. WT1, implicated in renin transcription, is a bioactive regulator of vessel pressure, while systemic uremia from metabolic byproducts creates a local cardiopulmonary environment conducive to inflammation with diminished healing $[9,34]$.

While classically catheterization is indicated in patients with acute coronary syndrome (ACS), it holds utility in revealing the degree of valvular and pulmonary decline in the young-adult populous suffering from systemic disease burden such as DDS, especially in the setting of hypertension. Oxygen flux has been shown to be a major predictor of long-term mortality in patients with heart failure secondary to hypertension, giving credence to an increased spotlight to cardiorenal syndrome in patients with a dysfunctional kidneys and concomitant dyspnea [35]. Moreover, the quick onset of five-months between an echocardiogram that showed trace elements of cardiovascular compromise and a study illustrating more evident cardiovascular compromise exemplifies the urgency that warrants vigilant cardiovascular monitoring in patients not only suffering from hypertension from DDS, but any intrinsic renal deficit.

\section{Conclusion}

Renal considerations of pulmonary hypertension cannot be ignored in the diagnosis of patients with signs of kidney disease presenting with dyspnea. Namely, Denys-Drash Syndrome (DDS) leading to chronic renal failure and subsequent pulmonary dysfunction. By exploring the pathways underlying DDS and understanding the dynamics of cardiorenal metabolism, we can bridge pathways that refine our understanding pulmonary manifestations resulting from cardiorenal syndrome. Audits of cardiovascular anatomy via imaging is vindicated in adults with a history of congenital nephropathy, holding value comparable to measurements of uremia and creatinine, and are valuable in supporting the diagnosis of pulmonary hypertension. Considerations of cardiorenal syndrome in pulmonary hypertension should follow, which can aide in defining the acuity of pathology, saving erroneous workup while maximizing resource allocations intended to improve cardiopulmonary fitness in these patients.

\section{Acknowledgement}

None.

\section{Human and Animal Rights and Informed Consent}

This manuscript does not contain any studies with human or animal subjects.

\section{Conflict of Interest}

No conflict of interest.

\section{References}

1. Ronco C, Peter McCullough, Stefan D Anker, Inder Anand, Nadia Aspromonte, et al. (2010) Cardio-renal syndromes: report from the consensus conference of the acute dialysis quality initiative. Eur Heart J 31(6): 703-711.

2. Rezk T, Helen J Lachmann, Marianna Fontana, Ana Martinez Naharro, Sajitha Sachchithanantham, et al. (2019) Cardiorenal AL amyloidosis: risk stratification and outcomes based upon cardiac and renal biomarkers. Br J Haematol 186(3): 460-470.

3. Caetano F, Sérgio Barra, Ana Faustino, Ana Botelho, Paula Mota, et al. (2014) Cardiorenal syndrome in acute heart failure: A vicious cycle? Rev Port Cardiol 33(3): 139-146.

4. Núñez J, Miñana G, Santas E, Bertomeu González V (2015) Cardiorenal syndrome in acute heart failure: revisiting paradigms. Rev Esp Cardiol (Engl Ed) 68(5): 426-435.

5. Harjutsalo V, Forsblom C, Groop PH (2011) Time trends in mortality in patients with type 1 diabetes: nationwide population-based cohort study. BMJ 343: d5364.

6. Oh J, Rainer Wunsch, Martin Turzer, Malte Bahner, Paolo Raggi, et al. (2002) Advanced coronary and carotid arteriopathy in young adults with childhood-onset chronic renal failure. Circulation 106(1): 100-105.

7. Shirai M, Hirotsugu Tsuchimochi, Hisashi Nagai, Emily Gray, James $T$ Pearson, et al. (2014) Pulmonary vascular tone is dependent on the central modulation of sympathetic nerve activity following chronic intermittent hypoxia. Basic Res Cardiol 109(5): 432.

8. Havakuk O, Rezkalla SH, Kloner RA (2017) The cardiovascular effects of cocaine. J Am Coll Cardiol 70(1): 101-113.

9. Thenappan T, Ormiston ML, Ryan JJ, Archer SL (2018) Pulmonary arterial hypertension: pathogenesis and clinical management. BMJ 360: j5492.

10. Kohler B, Catherine Pienkowski, Françoise Audran, Martine Delsol, Maite Tauber, et al. (2004) An N-terminal WT1 mutation (P181S) in an $\mathrm{XY}$ patient with ambiguous genitalia, normal testosterone production, absence of kidney disease and associated heart defect: enlarging the phenotypic spectrum of WT1 defects. Eur J Endocrinol 150(6): 825-830.

11. Ozdemir DD, Hohenstein P (2014) Wt1 in the kidney- a tale in mouse models. Pediatr Nephrol 29(4): 687-693.

12. Pelletier J, W Bruening, CE Kashtan, SM Mauer, JC Manivel, et al. (1991) Germline mutations in the Wilms' tumor suppressor gene are associated with abnormal urogenital development in Denys-Drash syndrome. Cell 67(2): 437-447.

13. Natoli TA, Jing Liu, Vera Eremina, Karen Hodgens, Cong Li, et al. (2002) A mutant form of the Wilms' tumor suppressor gene WT1 observed in Denys-Drash syndrome interferes with glomerular capillary development. J Am Soc Nephrol 13(8): 2058-2067.

14. Addi T, Dou L, Burtey S (2018) Tryptophan-derived uremic toxins and thrombosis in chronic kidney disease. Toxins 10(10): 412.

15. Pei J, Magdalena Harakalova, Hester den Ruijter, Gerard Pasterkamp, Dirk J Duncker, et al. (2017) Cardiorenal disease connection during post- 
menopause: The protective role of estrogen in uremic toxins induced microvascular dysfunction. Int J Cardiol 238: 22-30.

16. Kawar B, Ellam T, Jackson C, Kiely DG (2013) Pulmonary hypertension in renal disease: epidemiology, potential mechanisms and implications. Am J Nephrol 37(3): 281-290.

17. Zuo L, Chuang CC, Hemmelgarn BT, Best TM (2015) Heart failure with preserved ejection fraction: defining the function of ROS and NO. Journal of Applied Physiology 119:944-951.

18. Schmederer Z, Natale Rolim, T Scott Bowen, Axel Linke, Ulrik Wisloff, et al. (2018) Endothelial function is disturbed in a hypertensive diabetic animal model of HFpEF: Moderate continuous vs. high intensity interval training. Comparative Study 273: 147-154

19. Clementi A, Grazia Maria Virzì, Ching Yan Goh, Dinna N Cruz, Antonio Granata, et al. (2013) Cardiorenal syndrome type 4: a review. Cardiorenal med 3(1): 63-70.

20. Rangaswami J, Vivek Bhalla, John EA Blair, Tara I Chang, Salvatore Costa, et al. (2019) Cardiorenal syndrome: classification, pathophysiology, diagnosis, and treatment strategies: a scientific statement from the American Heart Association. Circulation 139(16): e840-e878.

21. Sata Y, Head GA, Denton K, May CN, Schlaich MP (2018) Role of the sympathetic nervous system and its modulation in renal hypertension. Front Med 5: 82

22. Frame A, Wainford R (2017) The role of the mechanosensitive renal sensory afferent nerve sympathoinhibitory reno-renal reflex in sympathetic outflow, natriuresis, and blood pressure regulation. The FASEB Journal 31: 718-718.

23. Kopp UC, Smith LA, DiBona GF (1985) Renorenal reflexes: neural components of ipsilateral and contralateral renal responses. American Journal of Physiology-Renal Physiology 249(4): F507-F517.

24. Okusa MD, Rosin DL, Tracey KJ (2017) Targeting neural reflex circuits in immunity to treat kidney disease. Nat Rev Nephrol 13(11): 669-680.

25. Vaillancourt M, Pamela Chia, Shervin Sarji, Jason Nguyen, Nir Hoftman, et al. (2017) Autonomic nervous system involvement in pulmonary arterial hypertension. Respir Res 18(1): 201.
26. Thenappan T, Chan SY, Weir EK (2018) Role of extracellular matrix in the pathogenesis of pulmonary arterial hypertension. Am J Physiol Heart Circ Physiol 315(5): H1322-H1331.

27. Noh MR, Jang HS, Kim J, Padanilam BJ (2020) Renal sympathetic nervederived signaling in acute and chronic kidney diseases. Int J Mol Sci 21(5): 1647.

28. Zhang YX, Ji Feng Li, Yuan Hua Yang, Zhen Guo Zhai, Song Gu, et al. (2018) Renin-angiotensin system regulates pulmonary arterial smooth muscle cell migration in chronic thromboembolic pulmonary hypertension. Am J Physiol Lung Cell Mol Physiol 314(2): L276-L286.

29. De Man FS, Ly Tu, M Louis Handoko, Silvia Rain, Gerrina Ruiter, et al (2012) Dysregulated renin-angiotensin-aldosterone system contributes to pulmonary arterial hypertension. Am J Respir Crit Care Med 186(8): 780-789.

30. Malikova E, Kristina Galkova, Peter Vavrinec, Diana Vavrincova Yaghi, Zuzana Kmecova, et al. (2016) Local and systemic renin-angiotensin system participates in cardiopulmonary-renal interactions in monocrotaline-induced pulmonary hypertension in the rat. Mol Cell Biochem 418(1-2): 147-157.

31. Patek CE, Stewart Fleming, Colin G Miles, Christopher O Bellamy, Michael Ladomery, et al. (2003) Murine Denys-Drash syndrome: evidence of podocyte de-differentiation and systemic mediation of glomerulosclerosis. Hum Mol Genet 12(18): 2379-2394.

32. Nis HNIS (2011) Healthcare cost and utilization project (HCUP).

33. Badesch DB, Gary E Raskob, C Greg Elliott, Abby M Krichman, Harrison W Farber, et al. (2010) Pulmonary arterial hypertension: baseline characteristics from the REVEAL Registry. Chest 137(2): 376-387.

34. Steege A, Michael Fähling, Alexander Paliege, Anja Bondke, Karin M Kirschner, et al. (2008) Wilms' tumor protein (-KTS) modulates renin gene transcription. Kidney international 74(4): 458-466.

35. Poggio R, Arazi HC, Giorgi M, Miriuka SG (2010) Prediction of severe cardiovascular events by $\mathrm{VE} / \mathrm{VCO}_{2}$ slope versus peak $\mathrm{VO}_{2}$ in systolic heart failure: a meta-analysis of the published literature. Am Heart J 160(6): 1004-1014. 